\title{
The Research of Render to Textures during Making Virtual Reality Based on Unity 3D Technology
}

\author{
Yang Kuang ${ }^{1, a}$, Jie Jiang ${ }^{2, b}$ \\ ${ }^{1}$ Institute of Education, Jiang Xi Science and Technology Normal University, NanChang, China \\ ${ }^{2}$ Institute of Education, Jiang Xi Science and Technology Normal University, NanChang, China \\ aemail: ky_super@163.com, bemail: janyjiang@163.com
}

Keywords: Virtual Reality; Unity 3d; Render to Textures

\begin{abstract}
In the virtual reality, render to textures technology was widely used in animation, model making software or engine both have texture baking function. The baking technology can preprocess the virtual reality scene model, the shadow information is rendered into a map in the engine, it can no longer carry too much light calculation during real-time rendering process, it save system resource, and can greatly improve the system operation efficiency and enhance the display effect. This paper mainly discusses the unity 3D engine based on 3D model map baking in the virtual reality.
\end{abstract}

\section{Introduction}

Material is the material texture, is that the model gives the surface vivid characteristics, reflected in the color of the object, transparency, reflection, refraction, self luminous and roughness characteristics; mapping is the two-dimensional picture attached to the 3D model by software calculation, the formation of surface details and structure.

Mapping baking technology is a kind of model preprocessing technique. It first used in the game and construction roaming animation, large 3D modeling software (such as 3DS MAX, Maya) support this technology, it is a kind of is rendered the illumination information into a mapping mode, and then with a light map application information to the scene model technology. After baking technology processing, light texture information of model and the original model integrate into a new texture mapping[2].

In scene rendering, it do not need to system resources to calculate light, it saves a lot of time for real-time calculation of real-time virtual reality system of light information, so the rendering speed increase. Because it need a certain amount of time to bake, so it is not significance for 3D static frame of minor, but this technology can greatly improve the operating efficiency of the system for dynamic 3D real-time system, it has the performance of the rendering effect and make the effect of virtual reality system close to the real the effect of the scene.

\section{Zoom Baked mapping}

In the Unity 3D, scene baking texture size is $1024 * 1024$, but it has possible that the 3D model current baking is relatively simple, it will waste of memory space if use more than $1024 * 1024$ to map. As shown below, this is a model of baking texture, as shown in Figure 1, the left lower end was not fully used, but when running the program it occupies system resources. 


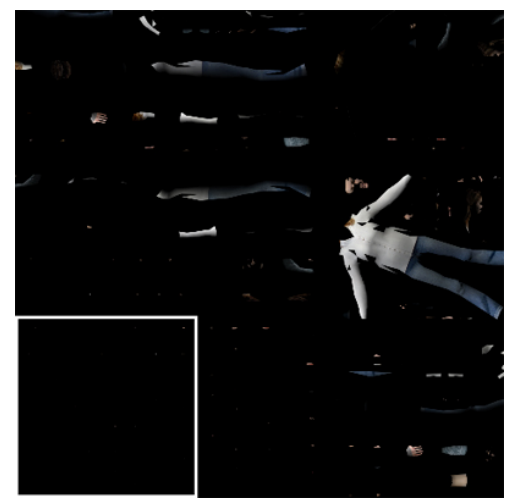

Fig.1. Model of Baking Texture

You can directly modify the baking texture size, but must repeated settings each time, and there will be problems in 3D model joints. We can use scripting to baking scene in 3D model, set baking texture size at the same time, solve the mapping problem of scale.

Bake () in unity 3D script represent the direct baking current scene, Clear () is to delete the current scene texture baking. Because the map size must be set to the power of 2, and is the best square textures, so baked mapping size set to $512 * 512$, this mapping space occupied $1 / 4$ of the original, which greatly save system resources, the code as follows:

[MenuItem ("shiyan/shiyan") ]

static void Init ( )

\{

LightmapEditorSettings.maxAtlasHeight $=512$;

LightmapEditorSettings.maxAtlasWidth $=512$;

Lightmapping.Clear ( );

Lightmapping.Bake ( );

\}n

\section{The Script with Baking Simulation to Generate Real-Time Shadow}

Set up a camera layer In the scene, set the camera will always move follow the moving of a 3D Model shadows. Operation process always maintain the side orthogonal shooting model, obtain the side of every frame image, and it will be rendered on the ground. In order to avoid the camera to get the background image, it will be set in the TransparentFX layer of the 3D models, the camera only intercept the layer model of each frame. Put the camera images of each frame of the capture of copy to the newly created RenderTexutre.

The code is below:

using UnityEngine;

using System.Collections;

public class NewBehaviourScript : MonoBehaviour \{

public RenderTexture s;

void Update（） \{

renderer.material.mainTexture $=\mathrm{s}$;

\}

void OnGUI ( )

\{

GUI.DrawTexture（new Rect（0，0，100，100), s );

\}

\section{The Factors Influencing the Baking Effect}

The greater the texture, the rendering effect is better. The size of generated texture baking 
directly affects the display effect. If set baking texture high, consumption is also high on the computer system resources, virtual reality system running speed will become slow. For complex scenes, it need for mapping baking planning. The surface area is large, complex models should give priority to the larger size of baking texture. Scene representation has little effect on the 3D model can be reduced when baked mapping accuracy. It can obtain high quality texture baking in saving system resources at the same time. In baking texture can also be obtained after the processing of its using image processing software, make further adjustments or modifications to the map.

Common mode has CompleteMap and LightingMap.CompleteMap is the fusion of 3D object original texture mapping and model generate the shadow map, generated maps containing the original texture and light information. It consumes high memory, can only be resolved after roasting map is not clear problem by increasing the map size, in order to achieve good results, you need a larger size. It suitable for small objects, and relatively high requirements for textured objects.

LightingMap can record the 3D object of diffuse reflection and projection effect. The texture is clear, but the light is weak, it suitable for a large-area brick wall, indoor and outdoor ground etc.. It is superimposed on the original map above, so the size can be set to a smaller size, the size of texture baking is generally not more than $256 * 256$.

Generally, a lot of softwares have the automatic deployment of UV mapping function in baked mapping. It is very convenient use automatic generation of UV map, but the effect is not always good, especially when a 3D object is rich in detail. Automatic unfolding of UV will have a lot of very small part of the area, and there are many spots. There are two ways to result these problems, one is to improve the automatic deployment of UV mapping, the angle of "threshold", there may be loss of details; the other is coordinate the UV texture for manual adjustment, this way consume more time. In the texture baking process, we only depends on the specific circumstances.

\section{Normal Mapping Combined with Baking}

The normal mapping does not really affect the same displacement, but the normal map can create geometry surface asperity model false image. The overall effect of the normal map is in the RGB channel, especially in the $\mathrm{R}$ and $\mathrm{G}$ channel, the two channel is often defined baking parameters $\mathrm{X}$ and $\mathrm{Y}$, this can make the low precision model rendering a high precision model of visual effect. As shown in Figure 2, a low precision 3D model and a high precision 3D model:

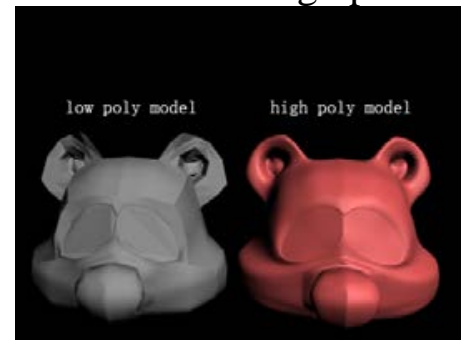

Fig.2. A Low Precision 3D Model and a High Precision 3D Model

In 3DS MAX, align two model to a position coordinate, bake the low precision of model, bake the high precision model details to low precision model texture, texture baking select normal maps (Normal map) mode, baked generated map as shown in Figure 3, which exaggerates the loss some white tick the part will have dark spots, need to be adjusted by texture mapping range, when processing is complete, low precision model with normal map rendering as shown in figure 4 .
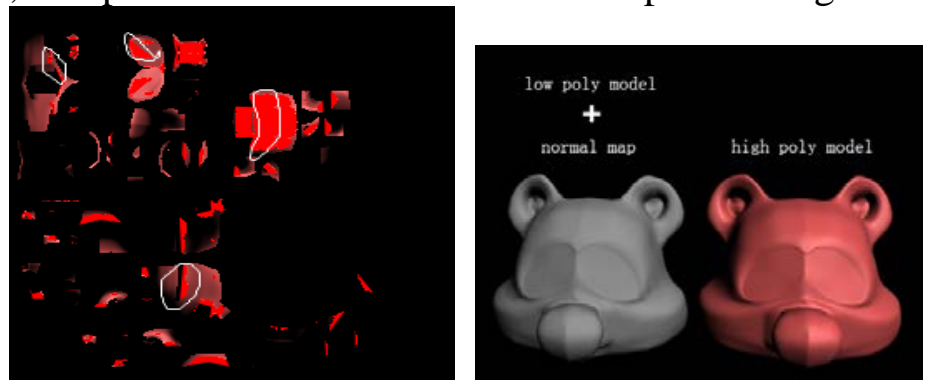

Fig.3. Baked Generated Map

Fig.4. Low Precision Model with Normal Map Rendering 
There are some differences between Normal mapping and bump mapping and displacement mapping. First of all, normal mapping has a color channel, different colors represent different surface texture information, and bump and displacement mapping only has gray channel, concave convex information in the gray channel storage; secondly, normal mapping and bump mapping belong to the same class map, use of color or gray information to simulate the bump model surface, produce the fluctuation effect. While the displacement mapping effectiveness number model surface, displacement mapping is generated bump effect through solid model; finally, the normal map not only record the bump (Bump) height information, direction information can also record the bump, so the normal map than bump effect to simulate more real, and render normal maps faster than rendering bump maps faster.

\section{Comparison of 3DS MAX and Untiy 3D in Texture Baking}

The final display effect between Unity 3D and 3DS MAX texture in baking is similar, but there also has some differences. Use What kind of baking depending on the particular situation. If want to acquire good effect, we should deal with texture.

The mapping and baking in unity 3d:(1)The parameter setting is less rich than the renderer plugin (Vray, Mental Ray) in 3DS MAX.(2)It only can bake light tracking effect, can not bake reflection.(3)Support model baking separately in different scenarios, the model can be used repeatedly.(4)Because each scene requires bake a group of maps, so if the number of scene is more, it will increase the number of textures.

The mapping and baking in 3DS MAX:(1)You can use the advanced render bake 3D models, such as Vray, Mental Ray etc.(2)There are some baking with reflection and refraction effect.(3)Because the light effect is fixed, so the engine which can only be set in accordance with the 3DS MAX, or the lighting effects will be wrong, baked models cannot be used in different scenes.

\section{Conclusion}

With the development of virtual reality technology, the real truth degree of the performance in virtual reality technology is highly valued by the industry. In virtual reality, use baked mapping approach to the performance of 3D model is becoming more and more common. At the same time, the 3D scene baking technology produced by the virtual reality can use lighting effects as a method for mapping the surface of the model, so that the virtual scene in virtual reality is more real and the scene file is more suitable for network communication. This article researchs the baking mapping technology in the unity 3D, and discuss the mapping and baking technology based on virtual reality technology, and make a summary on the use of texture in virtual reality baking.

\section{References}

[1] Chao Peng. Architectural animation secret 3dsmax production[M].BeiJing: Tsinghua University press,2011:7-8.

[2] Xinyou Cui, The application of texture baking technology in the city of 3D landscape system[J]. Science and technology information,2010(15):473-474. 